\title{
Estado del arte desde la comunicación sobre la violencia de género contra las mujeres migrantes jornaleras agrícolas abordada por otras ciencias
}

\section{State of the art from the communication on gender violence against women migrant farmworkers studied by other sciences}

\author{
Juan Ricardo Padilla Barrios* \\ Universidad Autónoma de Baja California \\ Avenida Álvaro Obregón s $\mathrm{n}$, Col. Nueva Mexicali, \\ C.P. 21100, Baja California, México
}

Editor: Rogelio del Prado Flores rpadilla@uabc.edu.mx

https://orcid.org/0000-0002-9009-1262

https://doi.org/10.36105/stx.2019n3.07

\section{RESUMEN}

T a violencia de género es una problemática mundial, social y vigente. Por lo que esta investigación se propone explorar la información de investigaciones empíricas internacionales sobre la violencia de género contra las mujeres. Presenta investigaciones empíricas centradas en artículos de bases de datos científicas de los últimos cinco años. En consecuencia, se contrastan objetivos generales, metodologías, resultados y referentes teóricos. Por consiguiente, se reconoce que las mujeres representan un grupo vulnerable, si se le agregan variantes como la migración y el desempeño del trabajo agrícola, se incrementa su condición, ya que viven en precariedad y desventaja social, al igual que sus pares varones, pero solo a ellas se agregan la violación, el hostigamiento y abuso sexual. Entonces, se requiere mayor explicación de las problemáticas de las mujeres para generar intervenciones desde la comunicación, con mayores competencias interpretativas y enfocados en la prevención que contribuyan a su erradicación.

\footnotetext{
* Licenciado en Ciencias de la Comunicación y Maestro en Administración por la Universidad Autónoma de Baja California (UABC). Cursa el Doctorado en Investigación de la comunicación por la Universidad Anáhuac México, Campus Norte. Profesor universitario de la UABC en las áreas de comunicación, ciencias administrativas y sociales. Certificado en Impartición de cursos de formación del capital humano de manera presencial grupal por el Consejo Nacional de Normalización y Certificación de Competencias Laborales (2017). Vicepresidente del Colegio de Comunicólogos de Ensenada (2017-2019). Presidente de la Academia de la Licenciatura en Ciencias de la Comunicación de la UABC (2018-2019).
} 
Palabras clave: violencia de género, violencia contra la mujer, jornaleras agrícolas, violencia sexual, violencia laboral.

\section{ABSTRACT}

Gender violence is a global, social and current issue. So, this research is based on the review of state of the art regarding gender violence against women. Presents empirical research focused on articles in scientific journals of the last five years. Consequently, general objectives, methodologies, results and theoretical references are contrasted. However, women represent a vulnerable group, if variants such as migration and the performance of agricultural work are added, their condition is increased, since they live in precariousness and social disadvantage, just like their male partners, but only women are raped, abused and sexually harassed. Therefore, a greater explanation of the problems of women is required to generate interventions from communication, with greater interpretative skills and focused on prevention, that contribute to their eradication.

Keywords: gender violence, violence against women, female farmworkers, sexual violence, workplace violence.

\section{INTRODUCCIÓN}

El siguiente estudio se concentra en explorar la información de investigaciones empíricas internacionales sobre la violencia de género contra las mujeres, producto de la revisión teórica centrada en artículos de los últimos años de bases datos científicas. Por lo que se analiza la violencia de género, en específico la sexual, laboral, institucional y comunitaria. Por otro lado, se examina la influencia del contexto sociocultural permeable a las féminas y la relación del apoyo social al afrontar estas problemáticas, y aunado a las repercusiones que enfrentan al ser violentadas. En paralelo, se exploran estudios sobre la migración y las formas de violencia que rodean a la mujer, los roles de género que experimentan modificaciones frente a la reconstrucción de su vida. También, se contrasta la violencia de género contra las mujeres en los ámbitos familiar, sexual e institucional, y cómo ellas perciben a las instituciones públicas encargadas de brindar seguridad y procuración de justicia. Por lo que los resultados demuestran que las mujeres reconocen la violencia contra ellas, pero en ocasiones lo dejan pasar para no generarse otros problemas. Por otro lado, la violencia persigue 
a las mujeres migrantes, en ocasiones ese es el motivo para salir de su lugar de origen, en el trascurso y hasta su asentamiento son blancos de violencia. Por otra parte, los roles sexuales de las mujeres tienden a modificarse a causa de la migración, ya que sus circunstancias varían alterando su realidad.

\section{ESTADO DEL ARTE}

\section{Violencia de género, contexto sociocultural y apoyo social}

Los textos empleados como punto de partida para esta investigación comparten un interés en investigar el entorno violento en el que se desenvuelven las mujeres jornaleras del noroeste de México. En los estudios de Aranda (2015), Aranda y Castro (2016) y Arellano (2015) se analizan las formas de violencia laboral contra mujeres jornaleras agrícolas, desde la perspectiva de jornaleras, en tres comunidades de Sinaloa, Sonora y Baja California. Sin embargo, Aranda (2015) tipificó la violencia desde la perspectiva de las mujeres, mientras que Aranda y Castro (2016) analizaron la vulnerabilidad de las jornaleras en su acceso a los servicios de salud y a la atención médica. A diferencia de Arellano (2015), quien buscó la relación entre la organización y la dinámica de las relaciones. Por su cuenta, Zúñiga (2018) analizó las condiciones sociales de precariedad y desventaja que viven las mujeres jornaleras en los ámbitos del trabajo, relaciones familiares y sentimentales para reflexionar sobre las formas en las que enfrentan la violencia.

Por el contrario, Acevedo (2015) estudió la percepción de la violencia laboral externa de las trabajadoras y las repercusiones en su salud para generar estrategias para enfrentarlas. Asimismo, Andrade-Rubio (2016) indagó en las condiciones en las que trabajan las mujeres migrantes en México para determinar la existencia de trata laboral y acoso sexual. No obstante, Flamtermesky (2014) analizó los principales protocolos y políticas de atención a mujeres víctimas de trata de personas, incluyendo la laboral y sexual, en Filipinas, Estados Unidos, Colombia y España para elaborar propuestas alternativas a las existentes, mientras García y Décosse (2014) se interesaron por el trabajo agrícola de migrantes en México y Francia por lo que analizan la Forma Migratoria de Trabajador Fronterizo (FMTF) y el contrato temporal Office des Migrations Internationales (OMI), con el fin de demostrar la lógica y la tendencia de las políticas migratorias en el ejercicio de la división internacional del trabajo. En cambio, Rivas, Panadero, Bonilla, Vásquez, y Vázquez (2018) analizaron el factor del apoyo social percibido, en cuanto a redes sociales, y la familia en mujeres víctimas de violencia de género en contextos de pobreza para identificar qué combinación de variables relacionadas con el apoyo social percibido permiten predecir el mantenimiento de la 
convivencia con el agresor. En cierta medida, Roca y Riccardi (2014) indagaron en la relevancia del apoyo social, en especial desde la funcionalidad familiar, en la evolución de daños.

Ahora bien, Aranda (2015), Aranda y Castro (2016), Arellano (2015),y Zúñiga (2018) aplicaron una metodología cualitativa a través de entrevistas grupales a 61 jornaleras y 18 entrevistas con expertos en el tema, tanto de la academia como del ámbito laboral, de Baja California, Sinaloa y Sonora. Del mismo modo, Andrade-Rubio (2016) aplicó la entrevista a profundidad a 20 jornaleras migrantes. Análogamente, García y Décosse (2014) efectuaron técnicas antropológicas para el trabajo de campo, así como la realización de encuestas a trabajadores agrícolas locales y foráneos (migrantes nacionales e internacionales), productores, técnicos y planeadores de los ingenios, además del registro audiovisual. A diferencia de Acevedo (2015), quien empleó una metodología cuantitativa mediante la aplicación de un cuestionario en entrevista personal a una muestra de 112 personas, paralelamente Rivas et al. (2018) aplicaron una entrevista estructurada a 136 mujeres víctimas de violencia de género en contextos de pobreza. Sin embargo, Roca y Riccardi (2014) emplearon un diseño de investigación mixto principalmente cuantitativo mediante entrevistas semiestructuradas. Si bien, Flamtermesky (2014) implementó una metodología de investigación-acción participativa feminista (IAPF) centrada en la inclusión de las mujeres estudiadas.

Por otra parte, los referentes teóricos del estudio de Aranda (2015) son Castro, 2012; Riquer y Castro, 2012; Velazco, 2007; Zúñiga, Bejarano, Scarone, Aranda y Arellano, 2012. Mientras que Aranda y Castro (2016) y Arellano (2015) se basaron en Bordieu, 2000; Bordieu, 2005; Wacquant, 2005. En cambio, Zúñiga (2018) y Arellano (2015) revisaron a De Grammont y Lara, 2005 y Lara, 2007, mientras que Zúñiga (2018) y Flamtermesky (2014) revisaron a Butler (2006). Por tanto, García y Décosse (2014) y Andrade-Rubio (2016) revisaron a Izcara (2010) e Izcara (2014). No obstante, García y Décosse (2014) revisaron a Castillo y Toussaint, 2010; García, 2013; Palacio, 2012. En cambio, Andrade-Rubio (2016) indagó en Cintas, 2011; Cueva y Terrón, 2014; Flamtermesky, 2014; García, 2014; Lara-Palacios, 2014; Mujica, 2014; Nejamkis y Castiglione, 2014. Por lo cual Rivas et al. (2018) leyeron a Bosch, Ferrer, Ferreiro, y Navarro, 2013; Cala, 2012; Cubells, Casamiglia, y Albertín, 2010; Vázquez, Panadero, y Rivas, 2015. A diferencia, Roca y Riccardi (2014) revisaron los trabajos de Ingram, 2011; Richards, 2011; Roy, 2011. Por la misma línea, Acevedo (2015) examinó a Martínez, 2011; Morales, 2009; Requena, 2008.

Por lo tanto, los trabajos de Aranda (2015), Aranda y Castro (2016), Arellano (2015) y Zúñiga (2018) reportan las desigualdades de poder entre hombres y mujeres manifiestas en las zonas agrícolas, lo que posibilita que se ejerza violencia contra las mujeres. Aranda (2015) clasificó la violencia contra las mujeres en tres entornos: la ejercida en los caminos 
o trayectos, en los hogares y la vinculada al crimen organizado. Asimismo, Zúñiga (2018) encontró que la totalidad de jornaleras entrevistadas perciben la violencia sexual, extrema y generalizada, dentro de su contexto laboral, siendo el acoso sexual el común denominador, igualmente Andrade-Rubio (2016) reportó que las mujeres solteras más jóvenes son las que lo padecen con mayor frecuencia. De igual forma, Arellano (2015) examinó las violaciones reconocidas por las mujeres (despido por embarazo, acoso sexual, frases denigrantes de contenido sexual, peticiones y exigencias de favores sexuales), minimizadas por las mismas mujeres para mantener un trabajo a pesar de las repercusiones en su entorno, también Aranda y Castro (2016) confirmaron la falta de servicios de salud y la precariedad en la que habitan las jornaleras.

De modo similar, Acevedo (2015) encontró que el tipo de agresión más frecuente es la verbal (gritos, insultos, groserías), seguido de la agresión física (empujones, golpes, armas de fuego) menos frecuente pero menos admisible y con mayores consecuencias físicas y psicológicas. Paralelamente, Rivas et al. (2018) demostraron que el apoyo social percibido representa un factor de auxilio frente a entornos desfavorables, entretanto las relaciones sociales no permitan la normalización y tolerancia de la violencia. En cambio, Roca y Riccardi (2014) afirman que el apoyo social percibido no predice la evolución clínica del paciente deprimido hospitalizado, en este caso un mujer violentada. Sino todo lo contrario, la funcionalidad familiar sí predice dicha evolución, ya que las personas con familias funcionales evolucionaban mejor, aunque no fueran percibidas como redes de apoyo socialmente efectivas.

En síntesis, existen diversos estudios que buscan mejorar las condiciones de vida de las mujeres a través del estudio de la violencia ejercida contra las mujeres según Aranda (2015), Aranda y Castro (2016), Arellano (2015), y Zúñiga (2018). Debido a la desigualdad entre los sexos, la generalidad de esta violencia resulta del producto de la práctica del abuso de poder de un hombre sobre los derechos humanos de las mujeres. La violencia contra las mujeres puede aparecer en cualquier estrato social, privilegiado o no, sin embargo, los contextos de pobreza, con presencia de adicciones y organizaciones delictivas, aumentan las posibilidades de que una mujer sea víctima de violencia de género (Aranda, 2015), donde el apoyo social se vuelve relevante (Rivas et al., 2018).

\section{MARCO TEÓRICO CONTEXTUAL}

\section{Roles de género, migración y violencia contra las mujeres}

El factor de la migración, aunado a la pobreza, dentro del perfil de las mujeres y los roles de género, extendidos en la familia, que conforman la identidad de la mujer son relevantes 
dentro de la violencia contra las mujeres. Al respecto, Ciurlo (2015) centró su interés investigativo en la migración femenina y los cambios en las relaciones de género en las familias, para ello identificó las disparidades de género y otras disparidades presentes antes de partir, así como también otras, el efecto mismo de la migración, en especial dentro de la familia. Del mismo modo, Willers (2016) estudió el vínculo relacionado entre la violencia y la migración de mujeres centroamericanas hacia Estados Unidos, desde la estrecha relación que guardan la pobreza y las formas de violencia. Igualmente, Posada, Mendoza, Orozco, Restrepo y Cano (2017) analizaron la determinación que implican los roles de género de las mujeres desplazadas, forzadas a abandonar su lugar de residencia, en relación con su salud.

Análogamente, Cárdenas, Terrón, y Monreal (2018) abordaron las afectaciones de los estereotipos a las mujeres migrantes mediante el análisis del proceso migratorio para demostrar si traía un cambio de roles en lo social y comunitario, e indagaron la exposición a los peligros durante su trayecto y sus formas de minimizarlos. De modo similar, Morad, Hamodi, Salazar, Rodriguez, y Jiménez (2019) estudiaron las construcciones socioculturales que reproducen la violencia dentro de las familias para identificar los significados sobre la masculinidad y feminidad, roles de género, asociadas a la violencia familiar por parte de agresoras y agresores.

Por tanto, Cárdenas et al. (2018), Morad et al. (2019), Posada et al. (2017) y Willers (2016) aplicaron una metodología cualitativa. Willers (2016) concretó entrevistas a $31 \mathrm{mu}$ jeres y tres hombres migrantes en diferentes etapas del proceso migratorio en Tijuana, Baja California, y Tapachula, Chiapas; asimismo, Posada et al. (2017) efectuaron 15 entrevistas semiestructuradas, seis entrevistas a profundidad y seis grupos focales con la participación de 49 mujeres en Medellín, Colombia; mientras que Cárdenas et al. (2018) aplicaron 26 entrevistas en profundidad a mujeres migrantes indocumentadas localizadas en los albergues de Tamaulipas, además de 12 entrevistas abiertas a informadores claves-profesionales de distintas dependencias gubernamentales y miembros de la sociedad civil; de modo similar, Morad et al. (2019) realizaron 19 entrevistas semiestructuradas a víctimas y agresores, analizadas de modo intra e intertextual. Por su parte, Ciurlo (2015) aplicó una investigación cuantitativa mediante un cuestionario estructurado, con una estrategia comparativa entre hombres y mujeres, aplicado a una muestra de 150 personas en los consulados de Roma y Milán con una proporción de mujeres del 64,7\% del total.

Por consiguiente, Ciurlo (2015) revisó a Anthias, 2000; Hondagneu-Sotelo, 1994; Morokvasic, 1984; Parella, 2005; Phizacklea, 1983; Scott, 1986. De la misma forma, Willers (2016) partió de los estudios de Bourgois, 2001; Menjívar, 2008; Scheper-Hughes y Bourgois, 2004. De modo similar, Posada et al. (2017) recuperaron la teoría de Gómez, 
Astaiza, y Minayo, 2008; Hernández y Gutiérrez, 2010; Strauss y Corbin, 2002. Igualmente, Cárdenas et al. (2018) examinaron a Ciurlo, 2015; García-Moreno, 2015; Martín, Martínez y Martínez, 2014. También, Morad et al. (2019) leyeron a Asturias, 2010; Bourdieu, 2000; Meler, 2013; Pineda y Otero, 2004; Serrano, 2015; Valcárcel, 2008.

De acuerdo con lo anterior, Willers (2016) encontró que la migración en ocasiones es una respuesta a la violencia social generalizada, en combinación con las desigualdades y la violencia de género, por lo que sus razones de migración son: la extorsión y amenaza de muerte por parte de los grupos delincuenciales, la necesidad de mantener a los hijos por ser madres solteras y la amenaza y violencia sufridas por parte de sus parejas masculinas. De igual forma, Ciurlo (2015) refirió que las causas para emigrar son múltiples, aunque la mayoría (46.2\%) están ligadas a factores de expulsión, ya que se trata, directa o indirectamente, de causas de tipo económico. De modo similar, Posada et al. (2017) demostraron que las mujeres se ven forzadas a cambiar su rol de cuidadora por el de trabajadoras, eso las lleva a trabajar largas jornadas de trabajo en condiciones inadecuadas que afectan su salud física y mental. Al mismo tiempo, Cárdenas et al. (2018) manifestaron que algunas mujeres inmigrantes que alcanzan un mayor poder adquisitivo contribuyen a un cambio de roles en la estructura familiar siendo las proveedoras primordiales de las familias. Paralelamente, Morad et al. (2019) descubrieron que las personas que viven en entornos violentos en la infancia tienden a normalizar estas conductas en la vida adulta, por lo tanto, al ser víctimas de violencia en la adultez les cuesta detectar la vulneración a sus derechos.

Para simplificar, no existe una única razón por la cual las mujeres salen de su casa y migran a otros lugares, pero al menos se reconocen tres principales: violencia por parte del crimen organizado, carencias económicas y violencia en el hogar (Ciurlo, 2015; Willers, 2016), es decir, mantienen el propósito de mejorar sus condiciones de vida. Las mujeres suelen cambiar de roles de género debido a las adversidades a las que se enfrentan (Posada et al., 2017), en algunos casos cambian de su rol de cuidadoras a uno de proveedoras (Cárdenas et al., 2018). En el transcurso de la migración pueden sufrir violencia, ya que su condición de vulnerabilidad la exponen constantemente, sin embargo, no en todos los casos pueden reconocerla, debido a que algunas mujeres que viven rodeadas de violencia tienden a normalizarla (Morad et al., 2019).

\section{MUJERES: VIOLENCIA SEXUAL Y GRADO DE APOYO INSTITUCIONAL}

La violencia sexual representa una de las problemáticas que vive la mujer como producto de la violencia de género, pero se agrava cuando una mujer violentada no recibe el apoyo 
esperado de las instituciones de seguridad pública, sino que se exponen a una violencia institucional. Por lo anterior, en España, Abril (2015) centró su investigación en analizar la complejidad de la comunicación en contextos de violencia de género, motivo por el que las instituciones requieren personal con competencias interpretativas para abonar a la minimización de una posible revictimización a causa de su condición de origen, raza, religión, etc. A la par, González y Garrido (2015) dedicaron su investigación a conocer la satisfacción de las mujeres víctimas de violencia de género con respecto a las medidas policiales de protección que reciben para establecer su eficiencia. Por su lado, en Argentina Carrington et al. (2019) indagaron cómo las Comisarías de la Mujer y la Familia (en adelante CFM) responden y trabajan con las comunidades locales y las juntas locales para prevenir la violencia de género, y qué aspectos podrían informar el desarrollo de nuevos enfoques para responder y prevenir la violencia de género en otras partes del mundo. A su vez, en México los estudios de Paredes, Llanes, Torres, y España (2016) buscaron demostrar la situación actual de la violencia de género ejercida en contra de las mujeres; a través de tres tipos, familiar o doméstica, institucional y feminicida para ofrecer un panorama más amplio de la problemática, mientras Espinoza y García (2018) exploraron los significados sexogenéricos de mujeres que sufren violencia de pareja, de varones que maltratan a sus parejas y de policías municipales que atienden dicha violencia.

De manera que, Espinoza y García (2018) y Paredes et al. (2016) se guiaron por una metodología cualitativa: Espinoza y García (2018) aplicaron entrevistas semiestructuradas a dos mujeres violentadas, un agresor y dos policías; Paredes et al. (2016), entrevistas y trabajo grupal con académicas, funcionarios públicos e integrantes de la sociedad civil que trabajan el tema de la violencia contra las mujeres. Por su lado, González y Garrido (2015) actualizaron de forma online un cuestionario de satisfacción y recopilaron la información de 1,128 víctimas de violencia de género, datos que fueron procesados mediante una metodología cuantitativa, al igual que Carrington et al. (2019), quienes la aplicaron a través de 51 entrevistas a empleados de cinco CMF. En cambio, Abril (2015) utilizó una metodología mixta centrada en grupos de discusión, encuestas y entrevistas, recabando impresiones de 600 profesionales de atención a víctimas, 27 intérpretes con experiencias y 12 supervivientes de violencia de género.

Por esta razón, Abril (2015) examinó a Cala, 2012; Del Pozo et al., 2014; Hale, 2011; Huelgo, 2006; López, 2007; Lorente, 2006; Molina, 2006; Mojica, 2014; Polzin, 2007; Toledano y Fernández, 2013. De modo similar, González y Garrido (2015) partieron de Alberdi, 2005; Cardozo, 1965; Marchal, 2010; Giese y Cote, 2000; Gómez, Muñoz, Vázquez, Gómez, y Mateos, 2012; López y Andrés-Pueyo, 2007; Muñoz et al., 2011; Pérez, Lozano, Gómez de Terreros y Aguilera, 2010; Zorrilla et al., 2010; Zurita, 2013. Por lo que 
Carrington et al. (2019) revisaron a Connell, 2007; Douglas, 2018; Goodman-Delahunty y Graham, 2011; Jubb et al., 2010; Loftus, 2008; Powell y Cauchi, 2013; Perovay Reynolds, 2017; Powell y Henry, 2018; Prenzler y Sinclair, 2013; Prokos y Padavic, 2002; Ragusa, 2013; Taylor et al., 2013; Voce y Boxall, 2018; Westera y Powell, 2017. Igualmente, Paredes et al. (2016) y Espinoza y García (2018) retomaron la teoría de Bourdieu, 2000. Del mismo modo, Paredes et al. (2016) revisaron a Arellano, 2008; Castro, 2006; Castro y Riquer, 2010; Frías, 2013; Galtung, 1969 y 1998; Moreno, 2009; Oliva, 2004; Paredes, 2005; Rosado, 2009; Villagómez, 2005. Igualmente, Espinoza y García (2018) partieron de Fondevila y Meneses, 2017; Gracia, Lila y García, 2009; Gutmann, 2000; Herrera, 2013; Johnson, 2005 y 2011; Logan, Shannon y Walker, 2006; López, Maier, Tarrés, y Zaremberg, 2014; López y Maier, 2014; Nuñez, 2011 y 2013; Torres, 2001; Zúñiga, 2015.

Por lo tanto, Abril (2015) resolvió que hay una demanda de mediación lingüística en todos los servicios públicos, misma que la mayoría de los profesionistas que los brindan aseguran una mejora significativa a la atención a las víctimas que no hablan el idioma oficial de la Administración, y puntualiza la necesidad de intérpretes especializados en contextos en violencia de género con el dominio de competencias específicas. Por su lado, González y Garrido (2015) encontraron un alto grado de satisfacción de las víctimas de violencia con la actuación policial, suponen resultados con medias superiores a ocho sobre diez en la mayoría de cuestiones, y $86.3 \%$ sí recomendaría los servicios policíacos a otra persona que pase por la misma situación. A lo que Carrington et al. (2019) reportaron que alrededor de $71 \%$ de los entrevistados de la CMF considera la prevención como parte fundamental de su trabajo, misma que obedece a tres estrategias principales: trabajar con mujeres para prevenir la revictimización; con la comunidad, para evitar que ocurra la violencia mediante la transformación de la cultura y las normas que sostienen la violencia contra las mujeres; así como con otras agencias para prevenir la violencia de género.

Paralelamente, Espinoza y García (2018) develaron que las mujeres reconocen la violencia a través de manifestaciones físicas y sexuales, pero identifican otras formas y modalidades; por lo que los oficiales mostraron centralidad en las agresiones físicas y sexuales para las intervenciones policiales, restando importancia a otras formas de violencia, mientras que el agresor reconoció que las agresiones a su esposa no coinciden con su percepción de su rol como hombre de familia. Mientras que Paredes et al. (2016) reconocieron al menos tres dimensiones dentro de la violencia institucional: es violencia directa porque un funcionario discrimina a una mujer por el hecho de serlo, es estructural porque la institución carece de mecanismos eficaces para evitar la discriminación, y es simbólica porque se legitima que ciertas personas sean valoradas de manera desigual en razón de ciertos marcadores sociales. 
En suma, las instituciones públicas carecen de intérpretes preparados en materia de violencia de género en contextos donde el idioma oficial no corresponde con el de las víctimas (Abril, 2015). A pesar de todo, en las agencias policiales españolas se perciben altos grados de satisfacción de los servicios de atención recibidos por las víctimas de violencia de género (González y Garrido, 2015); por otra parte, en Argentina se han ampliado esfuerzos por instalar comisarías atendidas, principalmente, por mujeres, para atender estos casos con mayor empatía, y mayormente enfocados a la prevención (Carrington et al., 2019). Asimismo, las mujeres víctimas de violencia, los agresores de la misma y los policías que atienden estos casos reconocen diversas formas de violencia, pero le dan mayor relevancia a la física y a la sexual por ser más evidente (Espinoza y García, 2018), por lo que las víctimas de violencia no son violentadas por un agresor primario, sino que continua al denunciarla ante un funcionario, ya que la revictimización, la discriminación y la desvalorización están presentes en las instituciones públicas de procuración de justicia (Paredes et al., 2016).

\section{METODOLOGÍA}

El presente estudio tiene por objetivo explorar la información de investigaciones empíricas internacionales sobre la violencia de género contra las mujeres, alojada dentro de las bases de datos científicas (EBSCO, Scopus y Google Académico). Por consiguiente, se examinaron artículos científicos provenientes de estas bases de datos presentados desde el 2015 a la fecha, utilizando las palabras clave: violencia de género, jornaleras agrícolas, violencia laboral, violencia sexual. Del mismo modo, se contrastaron los objetivos generales, la metodología, los referentes teóricos y los hallazgos. En consecuencia, los estudios analizados se insertan dentro de la piscología social, salud, sociología y traductología.

\section{RESULTADOS}

Las mujeres migrantes y jornaleras agrícolas reconocen la violencia que se ejerce contra ellas, presentada en diversas formas, es decir, están conscientes que a menudo las violentan (Aranda y Castro, 2016; Andrade-Rubio, 2016; Aranda, 2015; Arellano, 2015; Zúñiga, 2018). Sin embargo, lo admiten para no perder su trabajo o no generarse problemas adicionales (Arellano, 2015). Por lo tanto, ellas reconocen que las agresiones más comunes son el hostigamiento y acoso sexual (Arellano, 2015; Zúñiga, 2018), y que las más jóvenes las experimentan con mayor frecuencia (Andrade-Rubio, 2016). 
Por eso, al analizar la realidad de las mujeres se vuelve oportuno explorarla desde la categoría de la migración. Por lo que las causas de migrar pueden deberse a la extorsión o amenazas de parte del crimen organizado, la necesidad económica al ser madres solteras, o bien, amenazas y violencia sufrida por su pareja (Willers, 2016). No obstante, la mayoría de las causas son de orden económico-social (Ciurlo, 2015). En consecuencia, al provenir de entornos violentos, en ocasiones pueden no reconocer o tender a normalizar la violencia contra ellas en su condición de migrantes (Morad et al., 2019). Sin embargo, se adaptan al nuevo entorno para salir bien libradas, y en ocasiones, modifican sus preceptos de la feminidad (Cárdenas et al., 2018; Posada et al., 2017).

De manera que en España hay un pronóstico aceptable de la eficiencia con la que las autoridades atienden los temas de violencia contra las mujeres (Gonzáles y Garrido, 2015), pero se demandan traductores especialistas en violencia de género para las víctimas que no dominan el idioma oficial (Abril, 2015). Por otra parte, en Argentina se han obtenido buenos resultados de la implementación de comisarias especializadas atendidas por mujeres para tratar con mayor empatía a las víctimas de violencia de género (Carrington et al., 2019). Por otro lado, en México se reporta una mala praxis en la atención recibida por mujeres violentadas, se tiende a la revictimización, en ocasiones se les convence de retractarse o en todo caso no se le da seguimiento a su denuncia, y con ello se provoca mayor violencia de parte del agresor causada por la incompetencia de las autoridades policiales para atender y frenar esta problemática social (Espinoza y García, 2018; Paredes et al., 2016).

\section{ANÁLISIS Y DISCUSIÓN}

Mediante lo expuesto, se evidencia que las mujeres son conscientes de la violencia ejercida contra ellas, aunque lo dejan pasar para no generarse otros problemas. Ya que al denunciar una agresión se les visibiliza como víctimas, y eso resulta en una revictimización al denunciar, debido a los procedimientos rutinarios de las instituciones de justicia donde hacen que la víctima reviva lo sucedido en múltiples formas. Por otro lado, la familia, en particular la pareja, puede no tomarlo de la mejor manera y culpar a la mujer de lo acontecido, y con ello ser objeto de mayor violencia.

Por otro lado, la violencia que persigue a las mujeres migrantes, y en ocasiones ese es el motivo para salir de su lugar de origen, ya que las principales razones son del tipo económico-social. Por lo que pudieron haber experimentado violencia por parte de su pareja o familiares cercanos y con ello se busca una salida al huir a otro lugar. No obstante, en el trascurso pueden sufrir abusos, ya que carecen de protección siendo vulnerables a los ataques. 
Por otra parte, los roles sexuales de las mujeres tienden a modificarse a causa de la migración, ya que sus circunstancias lo exigen. Es decir, las mujeres con roles sexuales tradicionales, por llamarlos así, donde la mujer se encarga de la casa y el hombre de trabajar para proveer a los miembros de su familia, pueden verse afectados cuando la mujer trabaja y se vuelve proveedora igual que el hombre, lo que puede originar violencia contra la mujer. $\mathrm{O}$ bien, en ocasiones la mujer se vuelve la única proveedora, cuando el hombre no trabaja o es inexistente, por lo cual se reconfiguran los roles de acuerdo con las circunstancias de cada familia. Por ello, resulta interesante indagar en los cambios de sus representaciones sociales sobre el desempeño del hogar de una mujer trabajadora y encargada del sustento parcial o total de su familia.

\section{CONCLUSIONES}

La violencia de género es una problemática agravada para las mujeres. Por lo que se encuentra sustentada en los constructos sociales sobre la feminidad y la masculinidad, lo que repercute en las inequidades entre hombres y mujeres. Por consiguiente, las féminas gozan de menos privilegios, o derechos humanos básicos, a causa de cumplir normas morales, culturales, religiosas. Por ello, resulta alarmante que las disparidades se incrementan cuando el hombre, en su carácter de dominante, violenta a las mujeres provocándoles daños irreversibles, donde el mayor resulta con la muerte.

Por consiguiente, existe un vacío teórico dentro de los estudios de comunicación que aborden la violencia de género contra las mujeres desde los procesos de comunicación interpersonal, grupal e institucional. Es decir, se requieren investigaciones empíricas desde y con las mujeres afectadas, que se vinculen a los diversos interlocutores que generan la ruptura y detonan el conflicto. No obstante, los estudios encontrados de violencia de género desde la comunicación se centran en explicar las representaciones en el cine, la publicidad, fotografía, prensa, televisión, entre otras, pero no sobre el fenómeno en la realidad de la vida cotidiana en lo familiar, laboral y social. Por lo anterior, este trabajo investigativo retoma lo aportado por otras disciplinas, centrando la exploración en las interacciones, significados y procesos de los implicados para generar una aproximación al panorama comunicacional. 


\section{REFERENCIAS}

Acevedo, D. (2015). Violencia laboral externa. Sus expresiones y consecuencias en trabajadoras y trabajadores del sector salud. En Bustos, E., Caputo, M., Aranda, E. y Gimenez, M. (Ed.), Hostigamiento psicológico e institucional en Iberoamérica. Estado del arte y experiencias de intervención (pp. 13-22). Buenos Aires, Sb editorial. https://doi.org/10.26820/reciamuc/2.(3).septiembre.2018.406-417

Abril, M. I. (2015). La interpretación en contextos de violencia de género con referencia al caso español.Trans, (19.1), 77-94. https://doi.org/10.24310/trans.2015.v1i19.2093

Andrade-Rubio, K. L. (2016). Víctimas de trata: mujeres migrantes, trabajo agrario y acoso sexual en Tamaulipas. CienciaUAT, 11(1), 22. https://doi.org/10.29059/cienciauat.v11i1.654

Aranda, P. (2015). De espacios y violencias: vida cotidiana de jornaleras en comunidades del noroeste de México. Región y Sociedad, (662). https://doi.org/10.22198/rys.2014.0.a91

Aranda, P., y Castro, M. del C. (2016). El campo de la agroindustria en el noroeste de México y la salud de sus jornaleras: Una propuesta de estudio. Salud Colectiva, 12(1), 55-70. https://doi. org/10.18294/sc.2016.878

Arellano, M. del C. (2015). Violencia laboral contra jornaleras agrícolas en tres comunidades del noroeste de México. Región y Sociedad, (662). https://doi.org/10.22198/rys.2014.0.a90

Bustos, E.; Caputo, M.; Aranda, E.; Gimenez, M. (2015). Hostigamiento psicológico laboral e institucional en Iberoamérica : estado del arte y experiencias de intervención.

Cárdenas, R., Terrón, T., y Monreal, M. C. (2018). Redefinición de roles y relaciones de género en las migraciones internacionales. Estudio cualitativo en el Estado de Tamaulipas. Papeles de Población, 24(95), 153-179. https://doi.org/10.22185/24487147.2018.95.07

Carrington, K., Sozzo, M., Puyol, M. V., Gamboa, M., Guala, N., Ghilberto, L., y Zysman, D. (2019). El papel de las comisarías de la mujer en la ampliación del acceso a la justicia y la eliminación de la violencia de género. 63a periodo de sesiones de la Comisión de la Organización de Naciones Unidas sobre la condición jurídica y social de la mujer, sesiones de ONG, Nueva York. https:// doi.org/10.18356/dcfe304c-es

Ciurlo, A. (2015). La migración femenina y los cambios en las relaciones de género en las familias: el caso de las transmigrantes colombianas en Italia. Oasis, (21), 55. https://doi. org/10.18601/16577558.n21.04

Espinoza, C., y García, G. (2018). Meanings of gender and sexuality in partner violence: Victims, batterers and police officers in Hermosillo, Sonora. Estudios Sociologicos, 36(108), 571-594. https://doi.org/10.24201/es.2018v36n108.1602

Flamtermesky, H. (2014). Mujer frontera. Experiencia de investigación acción participativa feminista (IAPF) con mujeres víctimas de la trata de personas. Atbenea Digital, 14(4), 389-400.

García, M., y Décosse, F. (2014). Agricultura intensiva y políticas de migración laboral: Jornaleros centroamericanos en México y marroquíes en Francia. Migración y Desarrollo, 12(23), 39-65. https://doi.org/10.35533/myd.1223.mg.fd 
González, J. L., y Garrido, M. J. (2015). Satisfacción de las víctimas de violencia de género con la actuación policial en España. Validación del Sistema VioGen. Anuario de Psicologia Zuridica, 25(1), 29-38. https://doi.org/10.1016/j.apj.2015.02.003

Morad, P., Hamodi, C., Salazar, A., Rodriguez, M., y Jiménez, C. (2019). La violencia intrafamiliar con mirada de género. Pedagogia i Treball Social. Revista de Ciències Socials Aplicades, 8, 75-115.

Paredes, L., Llanes, R., Torres, N., y España, A. (2016). La violencia de género contra las mujeres en Yucatán. LiminaR. Estudios Sociales y Humanísticos, 14(2), 45-56. https://doi.org/10.29043/ liminar.v14i2.459

Posada, I., Mendoza, A., Orozco, I., Restrepo, C., y Cano, S. (2017). Roles de género y salud en mujeres desplazadas por la violencia en Medellín, 2013-2014. Ciencias de la Salud, 15(2), 189202. https://doi.org/10.12804/revistas.urosario.edu.co/revsalud/a.5754

Rivas, E., Panadero, S., Bonilla, E., Vásquez, R., y Vázquez, J. J. (2018). Influencia del apoyo social en el mantenimiento de la convivencia con el agresor en víctimas de violencia de género de León (Nicaragua). Informes Psicológicos, 18(1), 145-165. https://doi.org/10.18566/infpsic. $\mathrm{v} 18 \mathrm{n} 1 \mathrm{a} 08$

Roca, M., y Riccardi, A. (2014). Familia: Funcionalidad y efectividad como red de apoyo social en pacientes con depresión que están ingresados en el área de hospitalización de psiquiatría del hospital del IEss Dr. Teodoro Maldonado Carbo en la ciudad de Guayaquil. Informes Psicológicos, 14(2), 81-102. Recuperado de https://revistas.upb.edu.co/index.php/informespsicologicos/ article/view/5489/5092 https://doi.org/10.26820/recimundo/1.5.2017.194-212

Willers, S. (2016). Migración y violencia: las experiencias de mujeres migrantes centroamericanas en tránsito por México. Sociológica (México), 31(89), 163-195.

Zúñiga, M. (2018). Jornaleras agrícolas en el noroeste de México: transformaciones subjetivas en entornos de violencia. Sociológica (México), 33(94), 107-137. https://doi.org/10.22198/ rys.2014.0.a90 